\title{
Survivin expression may affect the neoadjuvant chemotherapy response in breast cancer patients
}

\author{
Sinan Demircioğlu ${ }^{1^{*}}$, Mehmet Artaç ${ }^{2}$, Levent Korkmaz ${ }^{2}$, Şeyda Gündüz ${ }^{3}$, Mustafa \\ Karaağaç $^{2}$, Hasan Esen ${ }^{4}$, Gülgün Erdoğan ${ }^{5}$, Zehra Er ${ }^{2}$, Elif Peştereli ${ }^{5}$, Lema Tavlı ${ }^{4}$, Hakan \\ Bozcuk $^{3}$
}

${ }^{1}$ Necmettin Erbakan University Meram Medical Faculty Department of Internal Medicine, Konya, Turkey

${ }^{2}$ Necmettin Erbakan University Meram Medical Faculty Department of Medical Oncology, Konya, Turkey

${ }^{3}$ Akdeniz. University Medical Faculty Department of Medical Oncology, Antalya, Turkey

${ }^{4}$ Necmettin Erbakan University Meram Medical Faculty Department of Pathology, Konya, Turkey

${ }^{5}$ Akdeniz University, Medical Faculty Department of Pathology, Antalya, Turkey

\begin{abstract}
To investigate whether there is a predictive effect of NF-kappaB, survivin, and Ki-67 expressions on pathological response and disease relapse in breast cancer $(\mathrm{BC})$ patients.

Ki-67, survivin and NF-kappaB expressions were analyzed in the pathology specimens of breast biopsy before and after neoadjuvant chemotherapy (NeoCT) in BC patients $(n=52)$. Event-free survival (EFS) (defined as recurrence or metastasis free) analyze was performed.

The median overall survival was 43.5 months and the median EFS was 51 months (95\% CI: 33.3-68.9) in all patients. The expression percentages of NF-kappaB, survivin, and Ki-67 significantly decreased after NeoCT $(p<0.001)$. Survivin expression level before NeoCT was significantly higher in patients who did not respond to NeoCT than both partial-responders and complete-responders $(p=0.038, p=0.010$, respectively). Type of NeoCT was the only independent factor on pathological response status $(p=0.007)$. Addition of taxanes to NeoCT improved pathological complete response rates about six times. However, no predictor was found to be a prognostic factor for EFS in multivariate analyze.

Higher survivin expression level before NeoCT may be associated with poor pathological response to NeoCT. These findings must be tested with prospective clinical trials.
\end{abstract}

Key Words: Breast cancer, Ki-67, neoadjuvant chemotherapy, NF-kappaB, pathological response, survivin

\section{Introduction}

Breast cancer $(\mathrm{BC})$ is the most common cancer in women and the second leading cause of death worldwide (1). Neoadjuvant chemotherapy (NeoCT) is recommended for stage II and III breast cancer. The aims of NeoCT in BC patients are: giving a chance of breast conserving surgery, providing operability of inoperable inflammatory disease, and prolonging the survival period. Pathological complete response (pCR) after $\mathrm{NeoCT}$ is related to longer survival in $\mathrm{BC}$ patients (2). Therefore, developing some parameters that may predict the pathological response to NeoCT is the subject of current researches.

NF-kappaB is a transcription factor whose expression level is associated with response to chemotherapy, resistance, and prognosis. NFkappaB suppression causes cell cycle arrest, apoptosis, and the inhibition of tumor proliferation (3). Furthermore, NF-kappaB inhibition sensitizes tumor cells to chemotherapy
(4). Hematopoiesis, transformation, apoptosis, proliferation, immunity, invasion, angiogenesis, and metastasis-related genes are kept under control by NF-kappaB. While NF-kappaB activation level is low in estrogen receptor (ER) $(+)$ patients, a higher level is detected in ER (-) patients. Thus, researchers believe that NFkappaB and ER inhibit the activity of each other (5). NF-kappaB activation is associated with resistance to various chemotherapeutic and endocrine agents, and it has been shown that inhibition of NF-kappaB increases cytotoxicity (6). In a study that investigated the role of NF-kappaB on locally advance $\mathrm{BC}$ patients, the NF-kappaB staining level was reduced significantly in patients who received anthracycline-based NeoCT (7). NFkappaB expression was found to be associated with ER negativity, high histological grade, high $\mathrm{Ki} 67$ index, and high pCR, but not associated with clinical response to NeoCT (8).

Survivin is a member of the antiapoptotic cellular system. It is responsible for cell division and the

*Corresponding Author: Sinan Demircioğlu, MD., Necmettin Erbakan University Meram Medical Faculty Department of Internal Medicine 42080, Konya-Turkey, Phone: +90 (332) 22364 34, Fax: +90 (332) 22378 49, E-mail: sinandemircioglumd@gmail.com 
inhibition of apoptosis (9). Transcriptional factors such as p53 can regulate survivin expression in various cancers. Correlation between expression of survivin and p53 accumulation has been detected in many cancers, especially of the gastric, pancreatic, prostate, lung, and squamous cellrelated varieties. Not only high survivin level is associated with resistance to both chemotherapy and radiotherapy, but also it is an indicator of poor prognosis (10). In a study that investigated survivin expression in a spectrum of benign to malignant lesions of the breast, the rate of survivin-positive staining cells was highest in highgrade ductal carcinoma in situ (DCIS) (95\%) (11). The researchers detected a trend toward a higher percentage of cells staining for survivin in BC cases that were ER (-), PR (-), or Her2/neu (+), although this was not statistically significant. In vitro studies have shown that survivin is upregulated by estrogen. Estrogen may affect cell survival through the upregulation of the antiapoptotic gene survivin (12).

$\mathrm{Ki}-67$ index is a cell proliferation marker. A low Ki-67 index is found to be a good prognostic parameter for both disease-free survival and overall survival in BC patients (13). The BC patients who have a high Ki-67 index respond well to initial chemotherapy, but those patients have a poor prognosis (14). Ki-67 index is a significant prognostic factor for neoadjuvant therapy (15).

Therefore, we aimed to investigate whether there is a predictive effect of NF-kappaB, survivin, and Ki-67 parameters on pathological response and disease relapse.

\section{Materials and Methods}

Eligibility: BC patients who received NeoCT and followed up between 2007 and 2014 in Necmettin Erbakan University (NEU) Meram Faculty of Medicine and Akdeniz University Faculty of Medicine were enrolled in this study. We detected 52 patients, 41 from Meram Faculty of Medicine and 11 from Akdeniz University Faculty of Medicine. The patients had no previous diagnosis of carcinoma and were without distant metastases at the time of diagnosis. Their pathology specimens of breast mass biopsy before and after NeoCT were included to this study. This study was approved by the Ethical Committee of Meram Faculty of Medicine in NEU and supported by the Scientific Research Projects (BAP) of NEU, by project number 121518025.

Response Evaluation: Response status was determined by FDA-approved pathological response criteria (16). Pathological complete response was defined to have no invasive residuals in the breast and axilla. Adjuvant therapy was administered based on established clinicopathological criteria at that time.

Immunohistochemistry: Pathology specimens before and after NeoCT were evaluated to determine the Ki-67, survivin, and NF-kappaB expressions by staining percentage. Three sections were taken from tumors of the specimens. Five- $\mu \mathrm{m}$ sections fixed to paraffin blocks with formalin were deparaffinized with xylene and alcohol and then saturated with $0.03 \%$ hydrogen peroxide. Sections were washed with tris-buffered $0.1 \%$ saline-Tween- 20 at $\mathrm{ph}=7.6$, and they were incubated for 16 hours in a dry air oven. The immunohistochemical method was performed by an automated immunostainer (Ventana BenchMark XT; USA). One of the three sections was prepared by the same method and stained by NF-kappaB/p65 rabbit antibody (C22B4, Cell Signalling). The second was stained by survivin Ab 17 antibody (B0579, Assaybiotech), and the third was stained by $\mathrm{Ki}-67$ antibody (C0290, Assaybiotech). The ultraView Universal DAB Detection Kit was used as the secondary antibody in the device. Stained sections were examined by the same pathologist. Ki-67, survivin, and NF-kappaB positive nuclei were counted in three areas selected at random, using a 20x objective. The Ki-67, survivin, and NF-kappaB labeling index was calculated as the number of immunoreactive nuclei per total number of cells and expressed as a percentage. Expressions were defined as expression-first for the expression value before NeoCT, expression-last for the expression value after $\mathrm{NeoCT}$ in surgical specimens, and expression-dif for the difference of expressions before and after NeoCT.

Statistical Analysis: While evaluating the data obtained in this study, Statistical Package Program for Social Sciences (SPSS Version 20) software was used for statistical analysis. Mean \pm standard deviation, median, and percentage (\%) values were found. A chisquare test was used for comparing the categorical data. A t-test was used to assess the difference of mean for non-categorical data. The comparisons between pathological responders (three factors) were performed by using a One-way ANOVA test. Finally, post-hoc analyses were done with Tukey's test.

Predictability of biomarkers on pathological response (non, partial or complete reponder groups) was analyzed with multinomial logistic regression with all likelihoods, which are ER/PR positivity, age, menopausal status, clinical stage at diagnosis, number of NeoCT cycles, and type of NeoCT. We can give only the value of the median 
Table 1. Demographic and clinicopathological features of the patients with EFS data

\begin{tabular}{|c|c|c|}
\hline Demographics & $\mathrm{n}(\%)$ & EFS months $(95 \% \mathrm{CI})$ \\
\hline All patients & $52(100)$ & $51(33.3-68.9)$ \\
\hline $\begin{array}{l}\text { Age: Median / Range } \\
\\
47 /(26-84)\end{array}$ & & \\
\hline $\begin{array}{l}\text { Life } \\
\text { Still living } \\
\text { Exitus } \\
\end{array}$ & $\begin{array}{l}37(71) \\
15(29) \\
\end{array}$ & \\
\hline $\begin{array}{l}\text { Disease relapse } \\
\text { Relapsed } \\
\text { Not-relapsed } \\
\end{array}$ & $\begin{array}{l}25(48) \\
27(52)\end{array}$ & \\
\hline $\begin{array}{l}\text { NeoCT cycles (median) } \\
\quad<6 \\
>6\end{array}$ & $\begin{array}{l}31(60) \\
21(40) \\
\end{array}$ & $\begin{array}{r}39.5(4.8-74.1) \\
54.1(28.8-79.5) \\
\end{array}$ \\
\hline $\begin{array}{l}\text { Menopausal } \\
\text { Pre- } \\
\text { Post- } \\
\end{array}$ & $\begin{array}{l}29(56) \\
23(44) \\
\end{array}$ & $\begin{array}{c}39.4 \\
51(28.1-74.0) \\
\end{array}$ \\
\hline $\begin{array}{l}\text { Stage } \\
\text { II } \\
\text { III }\end{array}$ & $\begin{array}{l}24(46.1) \\
28(53.9)\end{array}$ & $\begin{array}{c}54.1 \\
36.5(29.1-44.0)\end{array}$ \\
\hline $\begin{array}{l}\text { ER/PR } \\
\text { Positive } \\
\text { Negative } \\
\end{array}$ & $\begin{array}{l}33(63.4) \\
19(36.6) \\
\end{array}$ & $\begin{array}{c}\text { NR } \\
24(10.4-37.9) \\
\end{array}$ \\
\hline $\begin{array}{l}\text { C-erbB2 } \\
\text { Positive } \\
\text { Negative } \\
\end{array}$ & $\begin{array}{c}8(15) \\
44(85)\end{array}$ & $\begin{array}{c}\text { NR } \\
39.4(19.6-59.3) \\
\end{array}$ \\
\hline $\begin{array}{l}\text { Pathological response } \\
\text { pNR } \\
\text { pPR } \\
\text { pCR }\end{array}$ & $\begin{array}{l}20(38) \\
24(46) \\
8(16)\end{array}$ & $\begin{array}{c}24.1(0.0-49.2) \\
54.1(24.8-83.5) \\
\text { NR } \\
P=0.052\end{array}$ \\
\hline $\begin{array}{l}\text { Adjuvant RT } \\
\text { Received } \\
\text { Not-received }\end{array}$ & $\begin{array}{l}40(77) \\
12(23)\end{array}$ & $\begin{array}{c}54.1 \\
26.2(10.6-41.7) \\
P=0.028 \\
\end{array}$ \\
\hline $\begin{array}{l}\text { Tumor histology } \\
\text { IDC } \\
\text { Others }\end{array}$ & $\begin{array}{c}46(88.4) \\
6(11.6)\end{array}$ & $\begin{array}{c}51(31.2-70.9) \\
19.5(0-44) \\
\end{array}$ \\
\hline $\begin{array}{l}\text { Type of NeoCT } \\
\text { Anthracycline } \\
\text { Anthracycline+Taxane } \\
\text { Anthracycline+Taxane +Trastuzumab } \\
\text { Not-known }\end{array}$ & $\begin{array}{c}21(40) \\
23(44) \\
7(14) \\
1(2) \\
\end{array}$ & $\begin{array}{c}34.1(1.7-66.5)^{*} \\
54.1(24.9-83.3)^{*} \\
\text { NR } \\
\text { NR }\end{array}$ \\
\hline $\begin{array}{l}\text { Type of Surgery } \\
\text { Breast Conserving Surgery } \\
\text { Modified Radical Mastectomy }\end{array}$ & $\begin{array}{l}6(11.5) \\
46(88.5)\end{array}$ & $\begin{array}{c}\text { NR } \\
51(33.3-68.9)\end{array}$ \\
\hline
\end{tabular}

EFS, event free survival; ER, estragen receptor; PR, progesterone receptor; NeoCT, neoadjuvant chemotherapy; pNR, pathological non-response; pPR, pathological partial response; pCR, pathological complete response; RT, radiotherapy; IDC, invasive ductal carcinoma; others, non-lobuler and non-ductal tumors of breast.*Survival analyze was used between these two groups (CAF versus CAF+Docetaxel sequencely); NR, not-reached; N, number of patients; CI, confidence interval.

overall survival (OS), but we could not analyze the predictability of biomarkers on OS because of insufficient number of events. A Kaplan-Meier curve was used to calculate the event free survival (EFS). EFS was calculated by subtracting the dates: date of disease relapse and the date of last seen of the patients without relapse minus the date of diagnosis. Univariate and multivariate cox regression analyses were used to assess predictive effect of the expressions and the other factors on EFS. Tumor histology of 22 of 25 progressed patients was infiltrative ductal carcinoma, and we could find the grade status of 13 of 25 patients excluded from cox regression analysis. $p$ values $<0.05$ were considered statistically significant. 


\section{Results}

Demographics and clinicopathological features are defined in Table 1. All of the 52 patients received NeoCT, and almost all of them administered anthracycline containing regimens. Half of them received taxanes, and trastuzumab was combined in $14 \%$ of patients in the neoadjuvant setting. After NeoCT, eight $(16 \%)$ patients had a complete response, $24(46 \%)$ had a partial response and 20 (38\%) patients had no response in the pathology reports. Forty $(77 \%)$ patients underwent adjuvant radiotherapy. Twenty-two $(42.3 \%)$ patients received adjuvant chemotherapy. Half of the patients received adjuvant hormonal therapy. Though most common metastases were detected in bone $(40 \%)$, the liver $(27 \%)$, brain $(20 \%)$, and lung $(13 \%)$ metastases were also observed. At the time of statistical analyses, 27 $(52 \%)$ patients had still been living disease-free, 10 $(19 \%)$ patients had been living with disease, and 15 $(29 \%)$ patients were dead.

The expression percentages of NF-kappaB, survivin, and Ki-67 significantly decreased after NeoCT $(p<0.001)$ (Figure 1, Figure 2). First values and reductions in the expressions of three parameters (NF-kappaB, survivin, and Ki-67) were assessed in the association with the clinicopathological features (Table 2). Survivinfirst was significantly higher in the patients who did not respond to NeoCT than both partialresponders and complete-responders $(p=0.038$, $p=0.010$, respectively). Additionally, NF-kappaBdif and Ki-67-dif were significantly higher in the patients who had pCR $(p=0.043, \quad p=0.018$, respectively).

In univariate logistic regression analyses, type of NeoCT, survivin-first, and the clinical stage at diagnosis were significantly associated factors on the pathological response ( $\mathrm{p}$-values $=0.003,0.016$, and 0.034 , respectively). These three factors

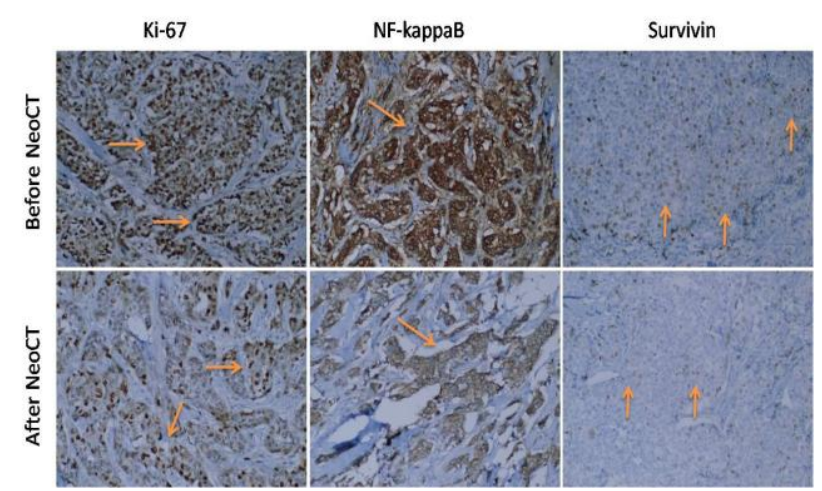

Fig. 1. NF-kappaB, survivin, and Ki-67 positive cells by immunohistochemistry (x100). entered multivariate logistic regression analysis. Type of NeoCT was the only independent factor in multivariate logistic regression analysis $(p=0.007)$. Addition of taxanes to NeoCT improved pCR rates about six times (Table 3). While response rate (total of pathologically complete and partial responses) was 33\% in patients who received anthracycline-based regimens, the rate was $80 \%$ in patients who received anthracycline plus taxane-based regimens $(p=0.001)$.

In this study, the median OS was 43.5 months and the median EFS was 51 months (95\% CI: 33.368.9). Adjuvant RT and hormonal treatment were the significant prognostic factors in the univariate cox regression analyses for EFS ( $p$-values $=0.033$ and 0.039 , respectively). When these two factors entered the multivariate cox regression model, none of them were found to be a prognostic factor (Table 4).

\section{Discussion}

Before neoadjuvant therapy, response prediction is very important for the selection of treatment modalities and can protect patients from unnecessary drug toxicities. Thus, we aimed to determine whether there is predictive effect of NF-kappaB, survivin, and Ki-67 parameters on pathological response and disease relapse. Survivin-first was significantly higher in the patients who did not respond to NeoCT compared to both partial-responders and complete-responders. In literature, pCR rates have been found to be about $10 \%$ to $60 \%$ in $\mathrm{BC}$ patients who undergo NeoCT. Higher pCR rates have been achieved with anthracycline plus taxane-based regimens. In our study, the rate of pCR was $15 \%$ and the addition of taxanes to NeoCT improved pCR rates significantly.

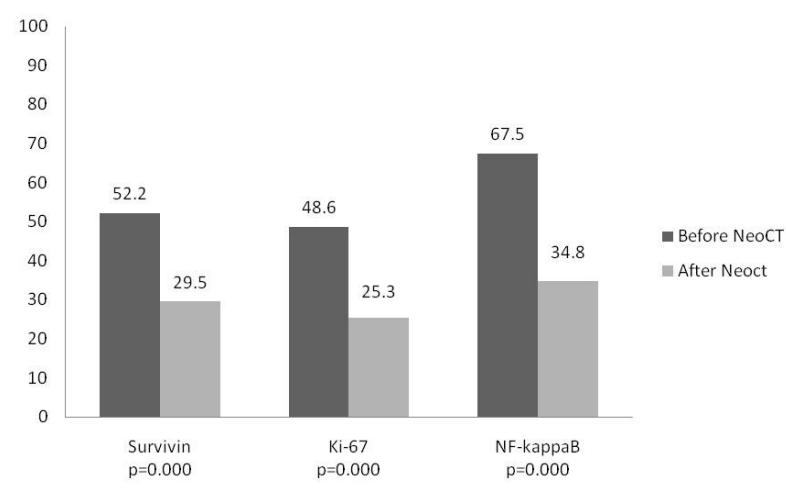

Fig. 2. Survivin, Ki-67 and NF-kappaB expressions (\%) before and after neoadjuvant chemotherapy. 
Table 2. Association between survivin, NF-kappaB, Ki-67 expressions before NeoCT, differences of expression and clinicopathological features

\begin{tabular}{|c|c|c|c|c|c|c|c|}
\hline Characteristic & $\begin{array}{c}\text { No. of } \\
\text { cases }\end{array}$ & $\begin{array}{c}\text { Survivin-first S } \\
(\%)\end{array}$ & $\begin{array}{c}\text { Survivin-dif } \\
(\%)\end{array}$ & $\begin{array}{c}\text { NF-kappaB-first } \\
(\%)\end{array}$ & $\begin{array}{c}\text { NF-kappaB-dif } \\
(\%)\end{array}$ & $\begin{array}{c}\text { Ki-67-first } \\
(\%)\end{array}$ & $\begin{array}{c}\text { Ki-67-dif } \\
(\%)\end{array}$ \\
\hline $\begin{aligned} \text { Age (year) } & \\
<47.5 & \\
\geq 47.5 & \\
& \text { p }\end{aligned}$ & $\begin{array}{l}29 \\
23\end{array}$ & $\begin{array}{l}57.7 \\
45.2 \\
0.157 \\
\end{array}$ & $\begin{array}{l}22.3 \\
23.2 \\
0.927\end{array}$ & $\begin{array}{c}72.7 \\
61.0 \\
0.127 \\
\end{array}$ & $\begin{array}{c}33.7 \\
31.4 \\
0.880 \\
\end{array}$ & $\begin{array}{c}55.5 \\
39.9 \\
0.293 \\
\end{array}$ & $\begin{array}{c}24.9 \\
21.3 \\
0.728 \\
\end{array}$ \\
\hline $\begin{array}{l}\text { Menopausal } \\
\text { status } \\
\text { Pre- } \\
\text { Post- }\end{array}$ & $\begin{array}{l}29 \\
23\end{array}$ & $\begin{array}{c}58.9 \\
43.7 \\
0.083\end{array}$ & $\begin{array}{l}24.0 \\
21.0 \\
0.749\end{array}$ & $\begin{array}{c}73.9 \\
59.5 \\
0.193\end{array}$ & $\begin{array}{c}34.7 \\
30.1 \\
0.762\end{array}$ & $\begin{array}{c}58.5 \\
36.2 \\
0.028\end{array}$ & $\begin{array}{c}27.1 \\
18.5 \\
0.414\end{array}$ \\
\hline $\begin{array}{l}\text { Stage } \\
\text { Stage II } \\
\text { Stage III } \\
\end{array}$ & $\begin{array}{l}24 \\
28\end{array}$ & $\begin{array}{l}58.7 \\
46.6 \\
0.172 \\
\end{array}$ & $\begin{array}{c}27.5 \\
18.5 \\
0.337\end{array}$ & $\begin{array}{c}75.7 \\
65.5 \\
0.160 \\
\end{array}$ & $\begin{array}{c}40.4 \\
26.1 \\
0.350\end{array}$ & $\begin{array}{c}60.5 \\
38.5 \\
0.029\end{array}$ & $\begin{array}{c}32.9 \\
15.1 \\
0.084\end{array}$ \\
\hline $\begin{array}{l}\text { ER status } \\
\text { positive } \\
\text { negative }\end{array}$ & $\begin{array}{l}33 \\
19\end{array}$ & $\begin{array}{c}53.3 \\
50.3 \\
0.723 \\
\end{array}$ & $\begin{array}{l}25.0 \\
18.7 \\
0.518 \\
\end{array}$ & $\begin{array}{c}65.6 \\
70.9 \\
0.643 \\
\end{array}$ & $\begin{array}{c}25.9 \\
44.5 \\
0.236 \\
\end{array}$ & $\begin{array}{c}41.8 \\
60.5 \\
0.075 \\
\end{array}$ & $\begin{array}{c}17.2 \\
33.9 \\
0.119 \\
\end{array}$ \\
\hline $\begin{array}{l}\text { PR status } \\
\text { positive } \\
\text { negative }\end{array}$ & $\begin{array}{l}26 \\
26\end{array}$ & $\begin{array}{c}47.2 \\
57.2 \\
0.256\end{array}$ & $\begin{array}{c}21.6 \\
23.8 \\
0.812\end{array}$ & $\begin{array}{c}69.5 \\
65.5 \\
0.716 \\
\end{array}$ & $\begin{array}{c}32.8 \\
32.6 \\
0.988\end{array}$ & $\begin{array}{c}41.2 \\
56.0 \\
0.146\end{array}$ & $\begin{array}{c}15.0 \\
31.6 \\
0.106\end{array}$ \\
\hline $\begin{array}{l}\text { C-erbB2 status } \\
\text { positive } \\
\text { negative } \\
\end{array}$ & $\begin{array}{l}21 \\
31\end{array}$ & $\begin{array}{c}45.3 \\
56.8 \\
0.199 \\
\end{array}$ & $\begin{array}{c}29.0 \\
18.4 \\
0.262 \\
\end{array}$ & $\begin{array}{c}69.8 \\
66.0 \\
0.733 \\
\end{array}$ & $\begin{array}{c}30.4 \\
34.2 \\
0.804 \\
\end{array}$ & $\begin{array}{c}37.3 \\
56.3 \\
0.066 \\
\end{array}$ & $\begin{array}{c}22.1 \\
24.1 \\
0.856 \\
\end{array}$ \\
\hline $\begin{array}{l}\text { Number of } \\
\text { NeoCT cycles } \\
\qquad 6.2 \\
>6.2 \\
\end{array}$ & $\begin{array}{l}31 \\
21\end{array}$ & $\begin{array}{c}52.3 \\
52.0 \\
0.980\end{array}$ & $\begin{array}{l}20.0 \\
26.6 \\
0.495\end{array}$ & $\begin{array}{l}64.9 \\
71.3 \\
0.557\end{array}$ & $\begin{array}{c}23.1 \\
46.9 \\
0.123\end{array}$ & $\begin{array}{c}36.8 \\
66.0 \\
0.004 \\
\end{array}$ & $\begin{array}{c}8.3 \\
45.5 \\
0.000\end{array}$ \\
\hline $\begin{array}{l}\text { Pathological } \\
\text { Response } \\
\text { status } \\
\text { pNR } \\
\text { pPR } \\
\text { pCR } \\
\end{array}$ & $\begin{array}{c}20 \\
24 \\
8\end{array}$ & $\begin{array}{c}66.1 \\
47.0 \\
33.1 \\
0.020\end{array}$ & $\begin{array}{c}14.6 \\
26.0 \\
33.1 \\
0.340\end{array}$ & $\begin{array}{l}78.7 \\
55.1 \\
76.8 \\
0.109\end{array}$ & $\begin{array}{l}40.4 \\
11.5 \\
76.8 \\
0.043\end{array}$ & $\begin{array}{c}51.6 \\
43.8 \\
55.7 \\
0.662\end{array}$ & $\begin{array}{c}13.2 \\
21.0 \\
55.7 \\
0.018\end{array}$ \\
\hline $\begin{array}{l}\text { Adjuvant RT } \\
\text { Received } \\
\text { Not-received } \\
\text { p }\end{array}$ & $\begin{array}{l}40 \\
12\end{array}$ & $\begin{array}{c}55.3 \\
42.0 \\
0.202 \\
\end{array}$ & $\begin{array}{c}24.5 \\
16.8 \\
0.220 \\
\end{array}$ & $\begin{array}{r}68.7 \\
63.5 \\
0.695 \\
\end{array}$ & $\begin{array}{r}36.0 \\
21.7 \\
0.430 \\
\end{array}$ & $\begin{array}{c}50.2 \\
43.5 \\
0.588 \\
\end{array}$ & $\begin{array}{c}25.1 \\
17.4 \\
0.534 \\
\end{array}$ \\
\hline $\begin{array}{l}\text { Grade status } \\
\text { Grade } 1 \\
\text { Grade } 2 \\
\text { Grade 3 }\end{array}$ & $\begin{array}{c}2 \\
21 \\
6\end{array}$ & $\begin{array}{l}23.0 \\
49.5 \\
63.0 \\
0.115\end{array}$ & $\begin{array}{c}23.0 \\
9.9 \\
39.3 \\
0.803\end{array}$ & $\begin{array}{l}72.5 \\
73.0 \\
40.0 \\
0.828\end{array}$ & $\begin{array}{l}-16.5 \\
39.8 \\
13.1 \\
0.246\end{array}$ & $\begin{array}{c}14.0 \\
40.3 \\
39.1 \\
0.202\end{array}$ & $\begin{array}{c}1.5 \\
15.1 \\
27.6 \\
0.412\end{array}$ \\
\hline $\begin{array}{l}\text { Tumor } \\
\text { histology } \\
\text { IDC } \\
\text { others } \\
\end{array}$ & $\begin{array}{c}46 \\
6\end{array}$ & $\begin{array}{c}51.2 \\
59.8 \\
0.535\end{array}$ & $\begin{array}{c}21.8 \\
29.8 \\
0.322\end{array}$ & $\begin{array}{c}64.3 \\
91.8 \\
0.000\end{array}$ & $\begin{array}{c}29.3 \\
58.3 \\
0.223\end{array}$ & $\begin{array}{c}47.0 \\
61.1 \\
0.379\end{array}$ & $\begin{array}{c}21.8 \\
34.5 \\
0.440\end{array}$ \\
\hline $\begin{array}{l}\text { *Comparing the } \\
\text { clinicopathologica } \\
\text { (pathological part } \\
\text { NeoCT, neoadjuv } \\
\text { complete response }\end{array}$ & the & apy; pNR, pa & thological $n$ & $\begin{array}{l}\text { response). ER, } \\
\text { esponse; pPR, } \\
\text { noma; others, }\end{array}$ & $\begin{array}{l}\text { gen receptor; P } \\
\text { logical partial re } \\
\text { obuler and non-d }\end{array}$ & $\begin{array}{l}\text { in } 52 \mathrm{BC} \\
\text { gical non-re } \\
\mathrm{R} \text {, progester } \\
\text { esponse; } \mathrm{pCR} \\
\text { tuktal tumors }\end{array}$ & $\begin{array}{l}\text { tients, the } \\
\text { onse), pPR } \\
\text { e receptor; } \\
\text { athological } \\
\text { breast. }\end{array}$ \\
\hline
\end{tabular}


Demircioğlu et al / Evolution of survivin expresion in breast cancer

Tablo 3. Univariate and multivariate logistic regression analysis of factors on pathological response

\begin{tabular}{|c|c|c|c|c|c|c|}
\hline \multicolumn{4}{|c|}{ Univariate } & \multicolumn{3}{|c|}{ Multivariate } \\
\hline Characteristic & Odds ratio & $95 \%$ CI & $\mathrm{P}$ & Odds ratio & $95 \% \mathrm{CI}$ & $\mathrm{p}$ \\
\hline Type of NeoCT* & 6.08 & $1.82-20.34$ & 0.003 & 6.22 & $1.60-23.6$ & 0.007 \\
\hline Survivin-first ${ }^{\beta}$ & 0.97 & $0.95-0.99$ & 0.016 & 2.25 & $0.59-8.50$ & 0.233 \\
\hline Clinical stage at diagnosis $\Omega$ & 3.54 & $1.09-11.46$ & 0.034 & 0.48 & $0.12-1.83$ & 0.286 \\
\hline NF-kappaB-first ${ }^{\beta}$ & 0.98 & $0.97-1.00$ & 0.112 & & & \\
\hline Number of NeoCT cycles & 1.13 & $0.93-1.50$ & 0.158 & & & \\
\hline Menopausal status & 1.85 & $0.58-5.87$ & 0.292 & & & \\
\hline Age & 1.01 & $0.97-1.06$ & 0.487 & & & \\
\hline Ki-67-first ${ }^{\beta}$ & 0.99 & $0.98-1.01$ & 0.64 & & & \\
\hline ER/PR status & 0.89 & $0.28-2.87$ & 0.855 & & & \\
\hline
\end{tabular}

${ }^{*}$ Taxane receiving positive or not. $\beta$ Lower or higher than median value of these expressions. $\Omega$ Stage 2 or 3 .

Tablo 4. Univariate and multivariate cox regression analysis for EFS

\begin{tabular}{|c|c|c|c|c|c|c|}
\hline \multicolumn{4}{|c|}{ Univariate } & \multicolumn{3}{|c|}{ Multivariate } \\
\hline Characteristics & HR & $95 \% \mathrm{CI}$ & $\mathrm{P}$ & HR & $95 \% \mathrm{CI}$ & $\mathrm{P}$ \\
\hline Adjuvant RT & 2.56 & $1.07-6.10$ & 0.033 & 0.46 & $0.19-1.12$ & 0.087 \\
\hline Adjuvant hormonal therapy & 2.40 & $1.04-5.52$ & 0.039 & 0.47 & $0.20-1.09$ & 0.081 \\
\hline Pathological response & 1.78 & $0.81-3.93$ & 0.148 & & & \\
\hline Type of NeoCT & 1.44 & $0.65-3.19$ & 0.360 & & & \\
\hline Survivin-first & 1.00 & $0.99-1.01$ & 0.443 & & & \\
\hline NF-kappaB-first & 0.99 & $0.98-1.00$ & 0.532 & & & \\
\hline Ki-67-first & 0.99 & $0.98-1.00$ & 0.804 & & & \\
\hline Menopausal status & 1.01 & $0.45-2.23$ & 0.973 & & & \\
\hline Age & 1.00 & $0.96-1.03$ & 0.939 & & & \\
\hline Clinical stage at diagnosis & 0.68 & $0.30-1.52$ & 0.347 & & & \\
\hline
\end{tabular}

EFS: event free survival. HR: hazald ratio.

It is well known that prognosis will be better in ER/PR (+) BC patients. However, ER (-) patients respond better to NeoCT (17). In our study, hormonal status did not affect pathological response. Furthermore, while NF-kappaB expression level is low in ER (+) patients, it is higher in ER (-) patients. Thus, it is assumed that NF-kappaB and ER inhibit the activity of each other (5). Another study investigated the activation of NF-kappaB in inflammatory and non-inflammatory $\mathrm{BC}$ patients with respect to ER and some other factors. NF-kappaB activation was higher in inflammatory $\mathrm{BC}$ and associated with a loss of ER (18). NF-kappaB expression levels were lower in ER (+) patients, but it was not statistically significant.

Higher Ki-67 level was found to be a predictive factor for clinical complete response, and higher levels also enhanced sensitivity to chemotherapy (14). Ki-67 expression level was not associated with pathological response in our study. In two other studies that evaluated the relationship between $\mathrm{Ki}-67$ expression and neoadjuvant therapy, Ki-67 expression level decreased with NeoCT $(19,20)$. In our study, Ki67 expression level decreased significantly in all groups of responders (complete, partial, and nonresponders). Lee and colleagues found posttreatment (neoadjuvant adriamycin plus docetaxel) $\mathrm{Ki}-67$ expression level to be a prognostic indicator for OS (21), but, it was not an indicator for EFS in our study. In another study, higher NF-kappaB expression was found to be associated with ER negativity, high histological grade, high Ki-67 index, and high pCR. However, NF-kappaB expression level was not a prognostic factor for clinical response to NeoCT (8). In our study, there was no correlation between these three expressions (NF-kappaB, survivin, and Ki-67).

NF-kappaB positivity before NeoCT was found to be a bad prognostic factor on clinical response in BC patients. While clinical response of positive patients was $20 \%$, it was $80 \%$ in negative patients. NF-kappaB nuclear staining was accepted as a predictive factor for resistance to NeoCT in $\mathrm{BC}$ patients (7). In another study, BC patients with 
negative tumor staining for NF-kappaB more commonly reached a pCR to NeoCT than those with positive tumor staining (22). However, we did not find any relationship between NF-kappaB expression levels and pathological response status $(7,22)$.

Tanaka and colleagues found high survivin expression in tumor to be an apoptosis inhibitor and a significant prognostic parameter of worse outcome in BC (23). In another study, BC patients with low survivin expression level showed significantly better disease-free survival (24). In our study, survivin expression rate (survivin-first) of non-responders was also significantly higher than responders.

Our study has some limitations; it is a retrospective study with a low number of patients. Predictability of biomarkers on OS will be analyzed in the future when we have an adequate number of death events.

In conclusion, Ki-67, survivin, and NF-kappaB expression levels decreased significantly in all patients after NeoCT, independent from pathological response status. Higher survivin expression level before NeoCT may be associated with poor pathological response to NeoCT. These findings must be tested with prospective clinical trials.

Conflict of Interest: None of the authors has any potential financial conflict of interest related to this manuscript.

Acknowledgements: This study was supported by the Scientific Research Projects (BAP) of NEU, by project number 121518025 .

\section{References}

1. Hidalgo JV, Bronsert P, Orlowska-Volk M, et al. Histological Analysis of $\gamma \delta \mathrm{T}$ Lymphocytes Infiltrating Human Triple-Negative Breast Carcinomas. Front Immunol 2014; 5: 632.

2. Connolly RM, Stearns V. Current approaches for neoadjuvant chemotherapy in breast cancer. Eur J Pharmacol 2013; 717: 58-66.

3. Bharti AC, Aggarwal BB. Nuclear factor-kappa B and cancer: its role in prevention and therapy. Biochem Pharmacol 2002; 64: 883-888.

4. Rundall BK, Denlinger CE, Jones DR. Combined histone deacetylase and NF-kappaB inhibition sensitizes non-small cell lung cancer to cell death. Surgery 2004; 136: 416-425.

5. Gionet N, Jansson D, Mader S, Pratt MA. NFkappaB and estrogen receptor alpha interactions: Differential function in estrogen receptornegative and -positive hormone-independent breast cancer cells. J Cell Biochem 2009; 107 : 448-459.

6. Das KC, White CW. Activation of NF-kappaB by antineoplastic agents. Role of protein kinase C. J Biol Chem 1997; 272: 14914-14920.

7. Montagut C, Tusquets I, Ferrer B, et al. Activation of nuclear factor-kappa B is linked to resistance to neoadjuvant chemotherapy in breast cancer patients. Endocr Relat Cancer 2006; 13: 607-616.

8. Jones RL, Rojo F, A'Hern R, et al. Nuclear NF$x \mathrm{~B} / \mathrm{p} 65$ expression and response to neoadjuvant chemotherapy in breast cancer. J Clin Pathol 2011; 64: 130-135.

9. Ambrosini G, Adida C, Altieri DC. A novel antiapoptosis gene, survivin, expressed in cancer and lymphoma. Nat Med 1997; 3: 917-921.

10. Sah NK, Khan Z, Khan GJ, Bisen PS. Structural, functional and therapeutic biology of survivin. Cancer Lett 2006; 244: 164-171.

11. Singh M, Bleile MJ, Shroyer AL, Heinz D, Jarboe EA, Shroyer KR. Analysis of survivin expression in a spectrum of benign to malignant lesions of the breast. Appl Immunohistochem Mol Morphol 2004; 12: 296-304.

12. Frasor J, Danes JM, Komm B, Chang KC, Lyttle CR, Katzenellenbogen BS. Profiling of estrogen up- and down-regulated gene expression in human breast cancer cells: insights into gene networks and pathways underlying estrogenic control of proliferation and cell phenotype. Endocrinology 2003; 144: 4562-4574.

13. Inwald EC, Klinkhammer-Schalke M, Hofstädter $\mathrm{F}$, et al. Ki-67 is a prognostic parameter in breast cancer patients: results of a large populationbased cohort of a cancer registry. Breast Cancer Res Treat 2013; 139: 539-552.

14. Petit T, Wilt M, Velten M, et al. Comparative value of tumour grade, hormonal receptors, $\mathrm{Ki}$ 67, HER-2 and topoisomerase II alpha status as predictive markers in breast cancer patients treated with neoadjuvant anthracycline-based chemotherapy. Eur J Cancer 2004; 40: 205-211.

15. Jones RL, Salter J, A'Hern R, et al. The prognostic significance of $\mathrm{Ki} 67$ before and after neoadjuvant chemotherapy in breast cancer. Breast Cancer Res Treat 2009; 116: 53-68.

16. Pathological Complete Response in Neoadjuvant Treatment of High-Risk Early-Stage Breast Cancer: Use as an Endpoint to Support Accelerated Approval [Internet]. Available from: http://www.fda.gov/downloads/drugs/guidance complianceregulatoryinformation/guidances/ucm 305501

17. Chávez-MacGregor M, González-Angulo AM. Breast cancer, neoadjuvant chemotherapy and residual disease. Clin Transl Oncol 2010; 12: 461 467.

18. Van Laere SJ, Van der Auwera I, Van den

East J Med Volume:22, Number:4, October-December/2017 
Eynden GG, et al. NF-kappaB activation in inflammatory breast cancer is associated with oestrogen receptor downregulation, secondary to EGFR and/or ErbB2 overexpression and MAPK hyperactivation. Br J Cancer 2007; 97: 659-669.

19. Burcombe RJ, Makris A, Richman PI, et al. Evaluation of ER, PgR, HER-2 and $\mathrm{Ki}-67$ as predictors of response to neoadjuvant anthracycline chemotherapy for operable breast cancer. Br J Cancer 2005; 92: 147-155.

20. Yin HF, Wang YH, Qin XQ, et al. [Effect of neoadjuvant chemotherapy on histologic grade and expression of biological markers in breast cancer]. [Article in Chinese]Zhonghua Zhong Liu Za Zhi 2009; 31: 858-862.

21. Lee J, Im YH, Lee SH, et al. Evaluation of ER and Ki-67 proliferation index as prognostic factors for survival following neoadjuvant chemotherapy with doxorubicin/docetaxel for locally advanced breast cancer. Cancer Chemother Pharmacol 2008; 61: 569-577.

22. Buchholz TA, Garg AK, Chakravarti N, et al. The nuclear transcription factor kappaB/bcl-2 pathway correlates with pathologic complete response to doxorubicin-based neoadjuvant chemotherapy in human breast cancer. Clin Cancer Res 2005; 11: 8398-8402.

23. Tanaka K, Iwamoto S, Gon G, Nohara T, Iwamoto M, Tanigawa N. Expression of survivin and its relationship to loss of apoptosis in breast carcinomas. Clin Cancer Res 2000; 6: 127-134.

24. Yamashita S, Masuda Y, Kurizaki T, et al. Survivin expression predicts early recurrence in early-stage breast cancer. Anticancer Res 2007; 27: 2803-2808. 